\title{
A portable double-slit quantum eraser with individual photons
}

\author{
T L Dimitrova ${ }^{1,3}$ and A Weis ${ }^{2}$ \\ ${ }^{1}$ Physics Faculty, University of Plovdiv 'Paissi Hilendarski', Tzar Assen Str. 24, \\ BG-4000 Plovdiv, Bulgaria \\ ${ }^{2}$ Physics Department, University of Fribourg, Chemin du Musée 3, CH-1700 Fribourg, \\ Switzerland \\ E-mail: doradimitrova@uni-plovdiv.bg
}

\begin{abstract}
The double-slit experiment has played an important role in physics, from supporting the wave theory of light, via the discussions of the wave-particle duality of light (and matter) to the foundations of modern quantum optics. Today it keeps playing an active role in the context of quantum optics experiments involving single photons. In this paper, we present a truly portable double-slit apparatus which demonstrates both the wave-particle duality of light and the phenomenon of quantum erasing. The device can be operated either with strong laser light and screen projection, or with individual photons, in which case quantum interference and quantum erasing are detected by a photomultiplier whose pulses are rendered acoustically by means of a loudspeaker. Alternatively, the phenomena can be displayed using multimedia projection of oscilloscope traces.
\end{abstract}

S. Online supplementary data available from stacks.iop.org/EJP/32/1535/mmedia

\section{Introduction}

In the early 19th century, double-slit interference was used to demonstrate the wave nature of light, based on the experiments by Thomas Young and their mathematical description by Augustin Fresnel. At the beginning of the 20th century, double-slit interference received renewed interest after Albert Einstein (and Max Planck) introduced a corpuscular theory of light by putting Newton's vision of light as a stream of particles on new theoretical and experimental grounds. The wave-particle duality of light and the particle-wave duality of

3 Author to whom any correspondence should be addressed. 
matter-introduced by Louis de Broglie-together with Heisenberg's uncertainly principle laid the foundations of early quantum mechanics.

The double-slit experiment played an important role in the Einstein-Bohr debate and at Solvay conferences, where it was an object of fiery discussions in the context of singlephoton interference. The discussion about the so-called which-way problem (destruction of interference by any attempt to measure the path/slit taken by the photon) led to the development of a quantum theory of the physical measurement process in which the conditions of the measurement itself influence the information retrieved from a quantum system.

For more than a century, Gedanken experiments based on the double slit (or more generally on two-path quantum interference) have been invaluable teaching tools for introducing basic and advanced concepts in quantum mechanics, such as the superposition principle, quantum interference, entanglement and quantum erasure. The double slit continues to be omnipresent in the contemporary scientific literature. Google, e.g., delivers more than 700000 hits when queried for 'double slit' (March 2011). This ongoing interest reflects Feynman's well-known statement that the double-slit experiment is 'a phenomenon which is impossible, absolutely impossible, to explain in any classical way, and which has in it the heart of quantum mechanics. In reality, it contains the only mystery' [1].

Triggered by the scarcity of commercial demonstration experiments in quantum optics, the authors have in the past developed different experimental setups that allow lecture hall demonstrations of the puzzling features of light seen as quantum mechanical objects $[2,3]$. These experiments are based on two-path interference in a Mach-Zehnder interferometer operated with individual photons from a strongly attenuated laser beam, together with photomultiplier (PM) detection. We have also realized a double-slit experiment with individual photon detection using an image-intensified CCD camera [2,3]. The former experiments have the drawback that convincing demonstrations require a heavy optical breadboard for mounting the interferometer, which makes the experiment transportable, rather than portable. The latter experiment is portable but necessitates an extremely costly [2] single-photon imaging camera.

The concept of quantum erasing (QE) was introduced by Scully and Drühl in 1982 [4]. A simplified version of QE with light has been discussed in detail by Kwiat and Hillmer [5], although their experimental implementation (diffraction by a thin wire) was done with strong light only. We have used two-path interference in a Mach-Zehnder interferometer [3]—and more recently in a Michelson interferometer-to demonstrate QE with individual photons. In these experiments, the two paths that each photon follows simultaneously (following the Copenhagen interpretation of quantum mechanics) are labelled by orthogonal polarizers and interference is destroyed in consequence. A third polarizer, inserted after the recombination of the two interfering paths, i.e. after the photon has left the interferometer, can erase the whichway information leading to a revival of the interference. The effect and its mathematical treatment in terms of single-photon entanglement are presented in [3, 6].

In this paper we present details of our recent development of a compact, truly portable quantum interference device, which allows demonstrations of the wave-particle duality and of $\mathrm{QE}$ of light. It is based on a double slit that can be illuminated with strong light or with individual photons. Wave interference is demonstrated by screen projection, while individualphoton interference and quantum erasure are demonstrated by moving a PM through the interference pattern. By rendering the PM pulses acoustically on a loudspeaker, interference is heard as a modulation of the photon click rate when the PM is moved through the diffraction pattern. 


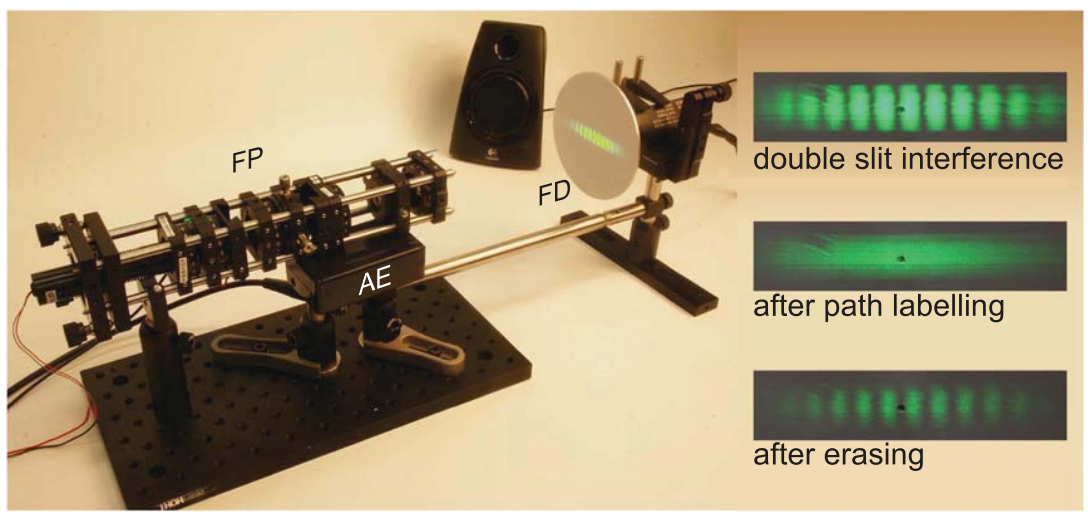

Figure 1. Left: assembled portable quantum eraser with optical microbench for projecting the double-slit interference pattern (fringe projector, FP), movable photomultiplier mounted behind projection screen with central aperture (fringe detector, FD) and loudspeaker. Laser and photomultiplier control electronics not shown. The angular encoder (AE) is a useful, but optional, feature. From top to bottom on the right: patterns recorded with strong light.

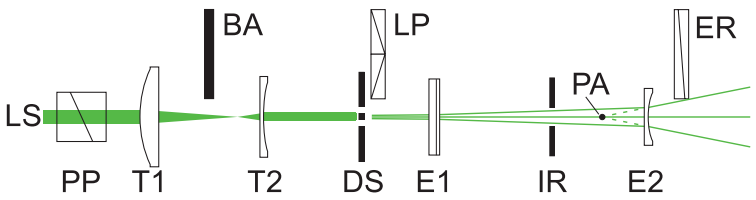

Figure 2. Schematic view of the double-slit fringe projector (FP). Elements on the optical axis are shown in top view. The components BA and LP can be inserted from the top. The eraser ER is inserted from the right end of the FP. The laser source (LS) is not shown.

\section{Double-slit quantum eraser: components and performance}

Figure 1 shows a photograph of the portable quantum eraser. Double-slit interference produced by a fringe projector (FP) is observed by a fringe detector (FD) consisting of a projection screen (wave interference) and a PM (particle interference). An optional angular encoder (AE) has proven to be a useful feature when using an oscilloscope (section 2.3) for the demonstrations.

\subsection{Fringe projector and classical interference}

The main optical components of the fringe projector (FP) are shown schematically in figure 2. A beam from a laser pointer (laser source, LS), linearly polarized at $45^{\circ}$ by a prepolarizer (PP) is compressed by a cylindrical telescope (T1, T2) in one dimension (perpendicular to the slits) and is diffracted by a double slit (DS). The interference fringes are projected onto a projection screen (PS) after magnification by two orthogonal cylindrical lenses (E1, E2). An iris diaphragm (IR) limits the visible pattern to the central fringes. The classical doubleslit interference pattern is seen on the projection screen in the top insert on the right of figure 1. 


\subsection{Acoustic demonstration of quantum interference}

In order to demonstrate quantum interference with individual photons, an attenuating neutral density filter (beam attenuator, BA) is inserted before the double slit. A PM, mounted behind a small aperture in the projection screen, detects individual photons [2, 3]. The PM 'looks' at the virtual image of the double slit through a narrow solid angle defined by a collimator, and the PM pulses are rendered acoustically by means of a loudspeaker. Double-slit interference with individual photons is demonstrated with the labelling polarizers (LP) removed. When rotating the PM assembly around a pivot axis (PA) near the virtual image of the double slit, one clearly hears a modulation of the click rate, as the aperture moves across the regions of constructive and destructive interference. The live acoustic perception of the interference pattern is impressive and demonstrates well the simultaneous observation of particle properties (PM clicks) and wave properties (constructive and destructive interference) of light.

\subsection{PM demonstration of quantum interference}

Our main objective was the development of a compact stand-alone apparatus with acoustic rendering of quantum interference and its destruction/revival by path-information labelling/erasing via audible clicks. Alternatively, the demonstrated effects can also be rendered in a visual manner by the (multimedia or direct vision) display of oscilloscope traces showing the corresponding individual PM pulses. In order to obtain a meaningful oscilloscope picture, one has to assure that the rotational scan of the PM occurs at a constant angular velocity. For this, we have equipped the device with a simple angular encoder that produces a dc voltage, $U_{\alpha}$, proportional to the PM's angular position $\alpha$ (details described in section 3). The encoder voltage and the analogue PM pulses are connected to two channels of a digital oscilloscope. The oscilloscope is operated in normal trigger mode and the trigger level is adjusted to a value of $U_{\alpha}$ corresponding to a PM position outside the fringe pattern. Scanning the PM across the fringe pattern then triggers both oscilloscope traces and the encoder voltage, i.e. the PM's position $\alpha$ is displayed together with the PM pulses. The encoder signal is useful for monitoring the smooth and uniform motion of the PM assembly across the interference fringes. It is also a useful feature for making quantitative measurements. Figure 3(a) shows the encoder voltage together with the PM pulses during a scan of the normal double-slit interference pattern. By inserting attenuators BA with different optical densities, one can show that the PM pulses pile up to yield a smooth (classical) interference pattern, similar to the one reported in our previous publication [3]. The demonstration described here is shown in the movie double-slit interference with waves and with particles, presented as supplemental material available at stacks.iop.org/EJP/32/1535/mmedia.

\subsection{Wave erasing and $Q E$}

Which-way labelling is achieved by inserting, immediately after the double slit, two orthogonally $(H / V)$ oriented linear polarizers. When these path-labelling polarizers (LP) are inserted, interference disappears, as can be seen well (figure 1, central insert on the right) with strong light (attenuator BA removed). Insertion of the erasing polarizer (ER) at the end of the FP (not shown in figure 1 ), oriented at $\pm 45^{\circ}$ makes interference reappear, however, with a diminished total intensity (bottom insert on the right of figure 1). The contrast of the interference pattern is seen to gradually diminish when ER is rotated away from the $\pm 45^{\circ}$ directions, and vanishes completely when ER is oriented along the $H$ or $V$ directions. Details on how the fringe pattern's intensity and contrast depend on the orientation of the ER were presented in our earlier publication [3]. We refer to this demonstration as wave erasing [3]. The 

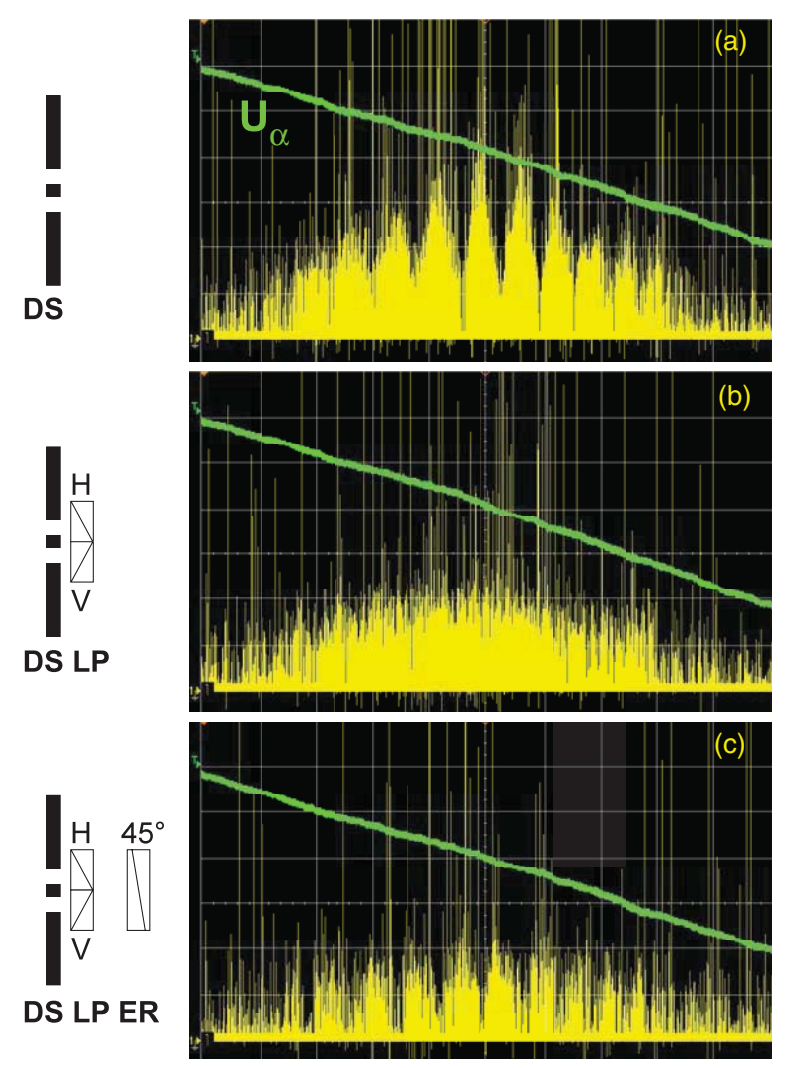

Figure 3. Oscilloscope traces of PM pulses and angular encoder voltage $U_{\alpha}$ during angular PM scan. From top to bottom: (a) fringe pattern from normal double slit, DS; (b) pattern with no interference when labelling polarizers, LP, are inserted; (c) revival of interference by insertion of the erasing polarizer, ER. All recordings are done with identical vertical sensitivity settings.

demonstrations described here are shown in the movie wave erasing in double-slit interference, presented as supplemental material available at stacks.iop.org/EJP/32/1535/mmedia.

QE can be observed in a similar way when the attenuator BA is inserted before the double slit. In this case, the acoustic signal reveals no audible modulation when the PM is moved across the fringe pattern. The corresponding oscilloscope trace shows no sign of interference, as can be seen in figure 3(b). Now inserting again the eraser, one hears and sees (figure 3(c)) a revival of the interference fringes, this time at the individual particle level. For a given laser power, one has to find the most appropriate attenuator BA such that the erased signal can be heard with a convincing contrast. In our apparatus, this is achieved with optical densities in the range of OD2-OD3. The demonstrations described here are shown in the movie quantum erasing in double-slit interference, presented as supplemental material available at stacks.iop.org/EJP/32/1535/mmedia.

The demonstration can also be made with the PM set to a fixed position. First the labelling polarizers LP are removed and the modulation of the click rate when the PM is moved through the fringe pattern is demonstrated. Next, the PM is positioned at the first (audible) minimum near the central bright fringe. Inserting the path-labelling polarizer will make the click rate go up, since adding which-way information kills (destructive) interference. Slipping on the 
erasing polarizer ER, preoriented at $+45^{\circ}$ (or $-45^{\circ}$ ) will then increase (or decrease) the click rate compared to the path-labelled configuration. This latter feature reflects the interchange of dark and bright fringes when the labelled beam is erased at $+45^{\circ}$ or $-45^{\circ}$, respectively $[3,5]$. A subsequent sweep of the PM convinces the audience that interference has been restored in the path-label-erased light beam.

\section{Construction of the quantum eraser}

In this section, we present technical details on the individual components of the fringe projector FP, the fringe detector FD and the angular encoder AE. All abbreviations refer to notations in figures 1 and 2. The appendix gives a list of all commercial components used and their suppliers.

\subsection{Fringe projector}

The fringe projector is based on Thorlabs' [7] $30 \mathrm{~mm}$ cage system, held by four 10" cage assembly rods, and mounted with a standard post and post holder onto a $300 \times 150 \mathrm{~mm}^{2}$ aluminium breadboard. The FP's purpose is to generate double-slit interference fringes with strong light and with individual photons.

3.1.1. Laser source (LS) and prepolarizer (PP). The light source is a $5 \mathrm{~mW}$ green $(\lambda=$ $532 \mathrm{~nm}$ ) laser module. The beam from most commercial laser pointers is neither parallel to its (cylindrical) case nor centred with respect to it. We found that mounting the laser module into a two-axis tilt mount for $1^{\prime \prime}$ optics offers sufficient freedom for aligning the device (see also section 3.1 .4 below). The laser module is held in the mount by slipping a $1^{\prime \prime}$ outer diameter O-ring over the module and squeezing the O-ring between two retaining rings in the tilt mount. We also found that the linear polarization of the laser module is not well defined, which leads to a poor contrast in the erased interference pattern. For this reason, we insert a linear prepolarizer PP, oriented at $45^{\circ}$, directly after the laser. In this way, one ensures equal intensities in the two paths when the $\mathrm{H} / \mathrm{V}$ path-labelling polarizers are inserted after the double slit. When mounting the laser module in its mount, one has to assure that its polarization is oriented along the prepolarizer's orientation.

3.1.2. Beam telescope (T1, T2). The circular laser beam profile is not well matched to the double-slit geometry. In order to optimize the intensity of the double-slit diffraction pattern, we opted for $\mathrm{a} \approx 5: 1$ compression of the beam in the direction perpendicular to the slits using two antireflection-coated cylindrical lenses ( $+50 \mathrm{~mm}$ plano-convex, $-9.7 \mathrm{~mm}$ plano-concave). Both lenses are mounted in cage rotation stages that allow adjusting their relative orientations and their orientation with respect to the slits. The lens T2 is glued onto a $1^{\prime \prime}$ diameter ring inserted into the (threaded) rotation stage.

3.1.3. Beam attenuator $(B A)$. For performing demonstrations with individual photons, the laser beam is attenuated before hitting the double slit. We perform this attenuation by inserting a suitable absorbing neutral density filter between the telescope lenses T1 and T2. We found that a filter with an optical density in the range OD2-OD3 is well suited for this purpose. These $1 / 2^{\prime \prime}$ diameter filters can be inserted in a repositionable way using Thorlabs' removable filter holder cage system. We have modified the cage plate as shown in figure 4(a) by mounting a micro-switch that is closed when the filter holder is inserted. The open/closed information 


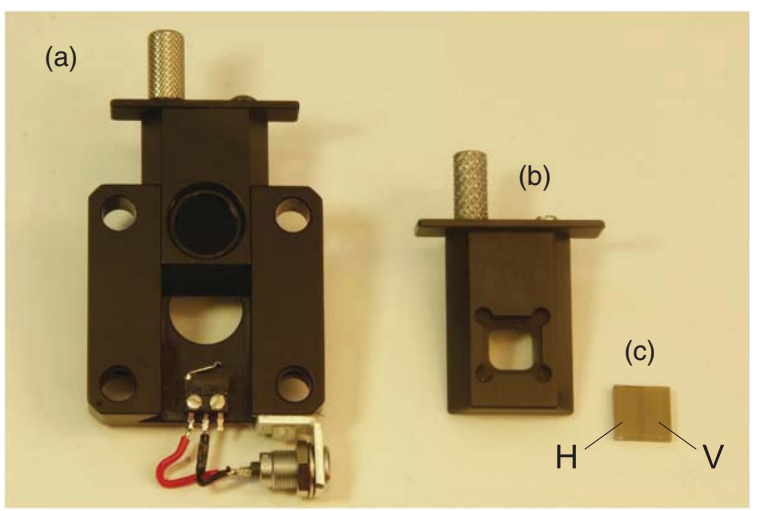

Figure 4. Details of system components: (a) removable beam attenuator BA and holder with a micro-switch for interlock control; (b) machined holder plate for a labelling polarizer; (c) labelling polarizers LP.

is transmitted via LEMO ${ }^{\circledR}$ cable to an interlock system that cuts the PM's high voltage supply when the filter is removed. In this way, irreversible damage to the PM is avoided if the attenuator is inadvertently removed while the PM is powered.

3.1.4. Double slit (DS). We use a commercial system consisting of two $40 \mu \mathrm{m}$ wide slits separated by $250 \mu \mathrm{m}$. We opted for this choice since it produces a reasonable number of bright central interference fringes. Pasco [8] supplies a set of four double slits that are electroformed on a single thin metal foil held between two glass cover slips in a photographic slide mount. We removed the metal foil from the slide, cut out the double slit of interest and taped it onto a $1 \mathrm{~cm}^{2}$ piece of microscope slide. That glass plate was then glued onto a suitable adapter ring held in an $x-y$ translation stage in such a way that the slide protrudes on the downstream side from the translator. The protrusion ensures that the labelling polarizer (LP) can be brought into close contact with the double slit. The translation stage, together with the laser tilt mount, can be used to centre the emerging diffraction pattern with respect to the system's optical axis and in particular with respect to the labelling polarizer that has no translational degree of freedom.

3.1.5. Labelling polarizer (LP). The path labelling polarizer, shown in figure $4(\mathrm{c})$, is a $10 \times$ $10 \mathrm{~mm}^{2}$ plate (custom manufactured by Codixx ${ }^{9}$ ) that consists of two juxtaposed dichroic glass polarizers that transmit $H$ - and $V$-polarized light, respectively. The $30 \mu \mathrm{m}$ wide slit dead zone between the two polarizers allows its relatively easy alignment with respect to the $210 \mu \mathrm{m}$ gap between the two slits using the double-slits' translation stage. The polarizer can be inserted in a repositionable way using Thorlabs' removable filter holder cage system. The split polarizer is mounted in a blank plate that is machined such that the polarizer can be glued/waxed into a square depression (figure 4(b)).

3.1.6. Beam expander (E1, E2, IR). A beam expander using two orthogonal cylindrical lenses allows independent adjustments of the vertical and horizontal extensions of the fringe pattern on the projection screen. We have opted for a $-25 \mathrm{~mm}$ lens (E1) for the vertical and a $-6.4 \mathrm{~mm}$ lens (E2) for the horizontal expansion, respectively. This choice presents a trade-off 


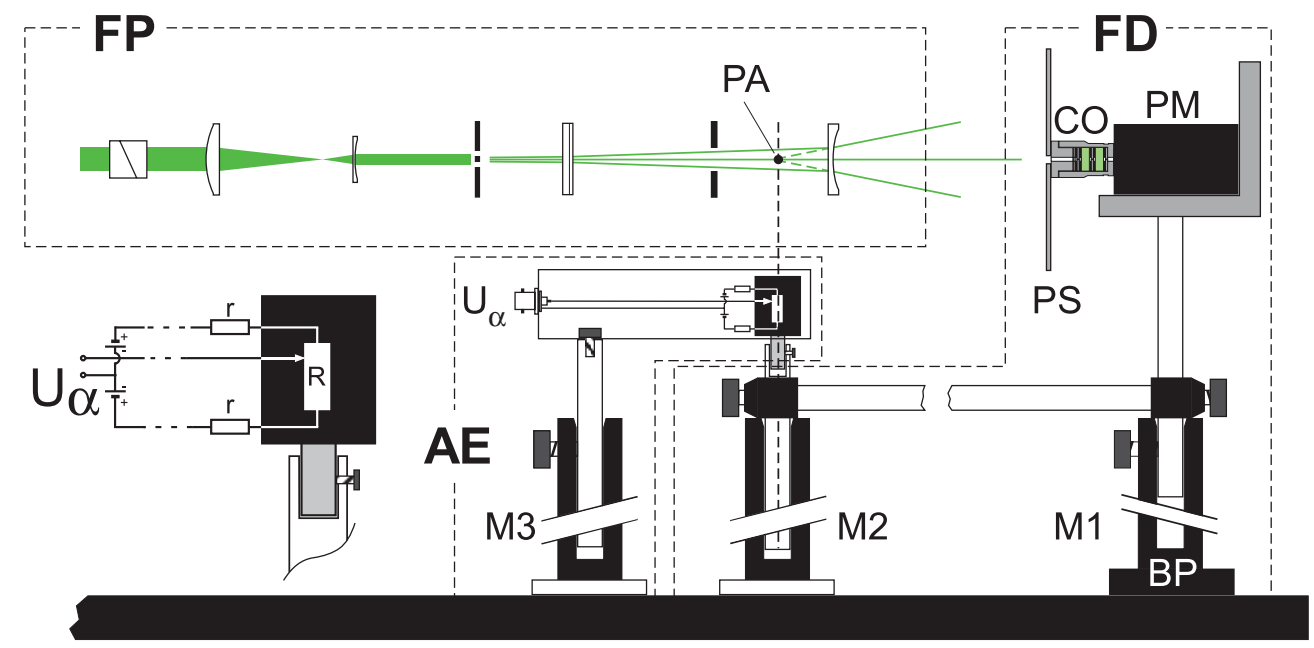

Figure 5. Side view of the fringe detector FD and angular encoder AE. The fringe projector FP is shown in top view.

between fringe pattern visibility and PM count rate under erasing conditions. Both lenses are mounted on rotation stages that allow adjustments of the fringe orientation. The lens E2 is glued onto a 1 " outer diameter ring that mounts onto the rotation stage.

An iris diaphragm is inserted between the lenses E1 and E2, in order to suppress the sideband fringes. In our experience, the revival of fringes due to single-slit diffraction complicates the discussion when presenting the device to a non-specialist audience. The iris serves another practical purpose: since the last cylindrical lens (E2) is only $6 \mathrm{~mm}$ wide, it leads to an unwanted cut and distortion of the outer regions of the complete fringe pattern. The iris suppresses this distortion.

3.1.7. Erasing polarizer (ER). The erasing polarizer is a $1 \mathrm{~cm}^{2}$ dichroic glass polarizer (manufactured by Codixx [9]) that is glued onto a $1^{\prime \prime}$ diameter mounting ring with a square depression, similar to the one in figure 4(b). The ring is held in a rotation stage used to adjust the eraser's orientation. Insertion of the ER (not shown in figure 1) is done by slipping it over the protruding ends of the mounting cage rods.

\subsection{Fringe detector (FD)}

Demonstrations with individual photons are made by moving a PM through the fringe pattern. The PM is forced to move on a circular path around the pivot axis PA, i.e. the virtual focus of the beam expander from which the diffracted beams seem to emerge. In this way, we perform experiments under normal, path-labelled and erased quantum interference conditions. A schematic view of the individual components of the fringe detector is shown in figure 5 .

3.2.1. Photomultiplier (PM). Individual photons are detected by a Hamamatsu H5784 photomultiplier ${ }^{4}$. The physics package of the PM module contains a high-voltage generator

${ }^{4}$ Hamamatsu's H5784 photomultiplier was recently superseded by model H10722-110 (3\% QE, \pm 5 VDC power supply). 


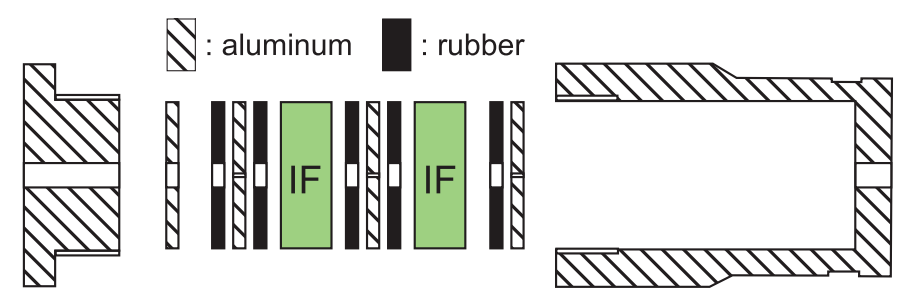

Figure 6. Exploded view of the diaphragms, spacers and interference filters (IF) in the collimator box $(\mathrm{CO})$ with screw-on cover.

and preamplifier, operated from a $\pm 15 \mathrm{~V}$ dc power supply. The high-voltage (PM gain) can be controlled either by an external potentiometer or by a variable (low) voltage. The PM has a quantum efficiency of $\approx 2 \%$ at $532 \mathrm{~nm}$. As discussed above, we use a micro-switch in the BA mount to ensure that high voltage is applied to the PM only when the light is strongly attenuated. The PM is mounted on a kinematic mount that allows tilt adjustments in two orthogonal directions, so that the PM's collimator always points towards the pivot axis during rotation.

3.2.2. Collimator (CO). The $\mathrm{PM}$ is made insensitive to perturbations from room lights by attaching it to a collimator consisting of three $300 \mu \mathrm{m}$ diameter diaphragms and two interference filters (transmission $\geqslant 45 \%$ within $532 \pm 5 \mathrm{~nm}$ ). The components are mounted inside an aluminium box (figure 6) that is fixed to the PM using a Hamamatsu adapter block. All components in the box are interspaced by $1 \mathrm{~mm}$ thick rubber discs with a central $2 \mathrm{~mm}$ diameter aperture to ensure light tightness. The PM thus 'sees' the outside world through the collimator in a solid angle that is equivalent to accepting light from a $15 \mathrm{~mm}$ diameter disc located $300 \mathrm{~mm}$ away. The collimator suppresses ambient light very efficiently so that the demonstrations can be made in a fully lit environment.

It is interesting to note that the interference filters also transmit the green spectral line of mercury emitted by conventional fluorescence tubes. A standard 'hearing photons' experiment that we perform is to remove the PM from its mount and to scan its line of sight by hand across individual (or rows of) fluorescence lamps in the lecture hall. Because of the small acceptance angle of the collimator, one can then 'hear' light from individual fluorescence tubes.

3.2.3. Projection screen (PS). The projection screen is a sandblasted aluminium disc, with a central $2 \mathrm{~mm}$ aperture, mounted on the collimator box.

3.2.4. Photomultiplier rotation system (PR). The PM tilt mount is fixed to a base plate BP by a standard post/postholder system (M1). A Teflon-coated tape glued to the bottom of the base plate minimizes friction with the table during PM motion. We ensure that during its motion, the PM's line of sight always points towards the virtual image of the slits-located between the two beam expansion lenses E1 and E2-by forcing the PM to move at a fixed radius from this image. The pivot axis PA is a Thorlabs' post that rotates freely in its post mount (M2), and the radius of rotation $(230 \mathrm{~mm}=$ pivot to $\mathrm{PM}$ photocathode distance $)$ is determined by a set of two rods that are tightly fixed to the pivot post and the PM carrying post. We found that the positioning of the pivot axis between the expansion lenses is not very critical. 


\subsection{Angular encoder (AE)}

For quantitative measurements, we have built an angular encoder that produces an analogue voltage, $U_{\alpha}$, proportional to the angular position of the PM. The pivot rod that defines the centre of rotation of the PM's motion is attached to a potentiometer that acts as a voltage divider. Symmetric voltages from two $1.5 \mathrm{~V}$ batteries are connected across the potentiometer and the voltage change at the potentiometer's cursor is then proportional to the angular position of the device. The potentiometer, batteries and a BNC connector are mounted in a component box (details shown on the lower left of figure 5), rigidly mounted (M3) on the breadboard.

\section{Summary and discussion}

Gedanken experiments have played —and continue to play—an important role for introducing the strange concepts of the quantum world. On the other hand, hands-on demonstration experiments have an undisputed value for convincing students and laymen audiences of the physical reality of abstract quantum concepts. However, quantum phenomena are often thought to occur at atomic and subatomic scales which, therefore, makes it difficult to devise sizable corresponding demonstration experiments.

Two-path interference of light quanta in a macroscopic experimental setup offers an elegant way for bridging these contradicting aspects. For instance, in recent years, we have developed several table-top experiments that illustrate the wave-particle duality (light detected as particles, but behaving as waves) and the phenomenon of quantum erasing, whose basis is single-photon entanglement (the intimate intrication of external and internal degrees of freedom of the photon). Our most thrilling demonstration consists in showing [10] how a (macroscopic) sheet of paper ${ }^{5}$, inserted by hand in one path of a two-path interferometer (adjusted to a fringe minimum), leads to a dramatic increase in the rate of detected photons, i.e. how the probability that a photon traverses an interferometer can be increased when forcing the photon to take a specific path, rather than offering the photon an alternative choice of two paths.

In this paper, we have presented details of the construction and performance of a compact $(3.5 \mathrm{~kg}$ ) demonstration experiment that illustrates the phenomena of quantum interference and quantum erasing. In its acoustic display mode, the experiment is well suited for small audiences that can gather around a table at exhibits, public events, student seminars or scientific conferences. The oscilloscope display mode can be used, together with multimedia projection, for demonstrations in larger lecture halls. The angular encoder feature makes the experiment also well suited for quantitative measurements in student laboratories.

A natural extension of the present apparatus would consist in introducing a translation stage operated blocking of either one of the slits, as we routinely do with the double-slit-CCD system discussed in section 1. During preparation of the present paper, we became aware that such a feature is implemented in a related commercial device (Teachspin's [11] two-slit interference, one photon at a time device).

We are aware that there is an ongoing debate-triggered by Lamb's article [12] on whether or not the photoelectric effect (used here to illustrate the particle aspect of light) truly demonstrates the corpuscular nature of light. For this reason, we have been cautious to speak of individual photons rather than single photons throughout the text. True heralded single-photon experiments may be realized by Bell state generators using

\footnotetext{
5 Blocking the beam by insertion of a hand would, of course, be an even more elegant demonstration of the effect. However, turbulence of the index of refraction of the air in the interferometer induced by the hand's heat radiation imbalances the interferometer and leads to poor results.
} 
Table A1. Commercial components used for building the portable quantum eraser: (a) double bored; (b) threaded; (c) $-9.7 \mathrm{~mm}$, plano-concave cylindrical; (d) $+50 \mathrm{~mm}$, plano-convex cylindrical; (e) $-6.4 \mathrm{~mm}$, plano-concave cylindrical; (f) $-9.7 \mathrm{~mm}$, plano-concave cylindrical; (g) $150 \times 300 \times 12.7 \mathrm{~mm}^{3}$; (h) $1 / 2^{\prime \prime}$ diameter, OD 2.0; (i) $1 / 2^{\prime \prime}$ diameter, OD 3.0.

\begin{tabular}{|c|c|c|c|c|}
\hline Component & Supplier & Product number & Units & Use \\
\hline Filter holder cage & Thorlabs [7] & CFH1 & 2 & BA, LP \\
\hline Blank plate & & CFH1-B & 1 & LP \\
\hline Extra filter holder & & CFH1-F & 1 & $\mathrm{BA}$ \\
\hline Cage alignment plate & & CPA1 & 1 & $\mathrm{FP}$ \\
\hline Cage rotation mount ${ }^{(a)}$ & & CRM1/M & 3 & $\mathrm{~T} 2, \mathrm{ER}, \mathrm{E} 2$ \\
\hline Cage rotation mount ${ }^{(b)}$ & & CRM1L/M & 2 & $\mathrm{~T} 1, \mathrm{E} 1$ \\
\hline Cage assembly rods & & ER10 & 4 & $\mathrm{FP}$ \\
\hline Cage $x y$ translator & & HPT1 & 1 & DS \\
\hline Kinematic cage mount ${ }^{(\mathrm{b})}$ & & $\mathrm{KC} 1-\mathrm{T} / \mathrm{M}$ & 1 & $\mathrm{LS}$ \\
\hline Cylindrical lens ${ }^{(\mathrm{c})}$ & & LC1054A & 1 & E1 \\
\hline Cylindrical lens $^{(\mathrm{d})}$ & & LJ1695RM-A & 1 & $\mathrm{~T} 1$ \\
\hline Cylindrical lens ${ }^{(\mathrm{e})}$ & & LK1087L2-A & 1 & $\mathrm{E} 2$ \\
\hline Cylindrical lens ${ }^{(\mathrm{f})}$ & & LK1836L1-A & 1 & $\mathrm{~T} 2$ \\
\hline Aluminium breadboard ${ }^{(\mathrm{g})}$ & & MB1090/M & 1 & $\mathrm{BP}$ \\
\hline Neutral density filter ${ }^{(h)}$ & & NE520B & 1 & $\mathrm{BA}$ \\
\hline Neutral density filter ${ }^{(i)}$ & & NE530B & 1 & $\mathrm{BA}$ \\
\hline Retaining ring & & SM1RR & 1 & $\mathrm{LS}$ \\
\hline Optical post & & TR40/M & 2 & $\mathrm{AE}$ \\
\hline Optical post & & TR50/M & 1 & $\mathrm{FP}$ \\
\hline Optical post & & TR200/M & 1 & $\mathrm{AE}$ \\
\hline Optical post & & TR75/M & 2 & $\mathrm{FD}, \mathrm{AE}$ \\
\hline Post holder & & $\mathrm{PH} 3 / \mathrm{M}$ & 1 & $\mathrm{FP}$ \\
\hline Post holder & & PH1.5/M & 1 & FD \\
\hline Post holder & & $\mathrm{PH} 1.5 \mathrm{E} / \mathrm{M}$ & 2 & $\mathrm{FD}, \mathrm{AE}$ \\
\hline Post end clamp & & RA180/M & 2 & FD \\
\hline Clamping fork & & CF125 & 2 & $\mathrm{FD}, \mathrm{AE}$ \\
\hline Cage plate adapter & & CP02B & 1 & $\mathrm{FP}$ \\
\hline Kinematic V-mount & & KM200 V/M & 1 & PM \\
\hline Double slit & Pasco [8] & OS1979 & 1 & DS \\
\hline Split polarizer & Codixx [9] & VIS 500 BC3 CW01 & 1 & LP \\
\hline Polarizer & & VIS 500 BC3 CW01 & 1 & ER \\
\hline Adapter block & Hamamatsu (see footnote 4) & A10030-01 & 1 & PM \\
\hline Photomultiplier ${ }^{10}$ & & H10722-110 & 1 & PM \\
\hline Green laser module & Roithner [14] & RLDD532-5-3 & 1 & LS \\
\hline Interference filters & Edmund [15] & NT43-070 & 2 & $\mathrm{CO}$ \\
\hline
\end{tabular}

parametric down-conversion that produces correlated (entangled) photon pairs. In the context of teaching, such experiments are described by Walborn et al [13], who discuss the historical development of quantum mechanical ideas, from wave-particle duality via the uncertainty principle and the quantum measurement problem to quantum erasing as a manifestation of quantum entanglement. However, we feel that our approach has its own didactical merits, since the concept of Bell states is a more advanced topic in quantum 
mechanics, whose conceptual understanding will definitely profit from the experiments shown here.

\section{Acknowledgments}

This project was supported in part by a Scopes grant (IZ73Z0_127942/1) from the Swiss National Science Foundation. One of us (TLD) acknowledges financial support from the LiMat collaboration and the Physics Department of the University of Fribourg. We appreciate the help of G Monney for designing the fringe projection optics. The authors thank Z Grujic for valuable help in the preparation of the video movies as well as P Knowles and N Castagna for the careful proofreading of the manuscript.

\section{Appendix. Component supplier details}

Table A1 lists all commercial components of the portable quantum eraser. Additional power supplies and controls for the laser and photomultiplier are needed for operating the device. Several adapter components (mainly $1^{\prime \prime}$ diameter rings) and some modifications to the commercial parts (mentioned in the text) were manufactured by the mechanical workshop at the authors' institute. The oscilloscope is not compulsory for the demonstrations, since the signal rendering by active loudspeakers gives satisfactory audible results.

\section{References}

[1] Feynman R P, Leighton R B and Sands M 1965 The Feynman Lectures on Physics vol III (Reading, MA: Addison-Wesley)

[2] Dimitrova T L and Weis A 2008 The wave-particle duality of light: a demonstration experiment Am. J. Phys. 76 137-42

[3] Dimitrova T L and Weis 2010 Single photon quantum erasing: a demonstration experiment Eur. $J$. Phys. 31 625-37

[4] Scully M O and Drühl K 1982 Quantum eraser: a proposed photon correlation experiment concerning observation and 'delayed choice' in quantum mechanics Phys. Rev. A 25 2208-13

[5] Hillmer R and Kwiat P 2007 A do-it-yourself quantum eraser Sci. Am. 296 90-5

[6] Ferrari C and Braunecker B 2010 Entanglement, which-way measurements, and a quantum erasure Am. $J$. Phys. $78792-5$

[7] Thorlabs http://www.thorlabs.com

[8] Pasco, http://www.pasco.com

[9] Codixx AG http://www.codixx.de/

[10] Weis A and Dimitrova T L 2009 Wave-particle duality of light for the classroom SPS-Mitteilungen http://www.sps.ch/artikel/progresses/wave particle duality of light for the classroom 13/

[11] Teachspin, http://www.teachspin.com

[12] Lamb W E Jr 1995 Anti-photon Appl. Phys. B 60 77-84

[13] Walborn S P, Terra Cunha M O, Pádua S and Monken C H 2002 A double-slit quantum eraser Phys. Rev. A 650338181

[14] Roithner Lasertechnik GmbH, http://www.roithner-laser.com

[15] Edmund Optics, http://www.edmundoptics.com/ 